\title{
О ФУРЬЕ-СПЕКТРАХ ЯМР ЖИДКОСТЕЙ, ПОЛУЧЕННЫХ В УСЛОВИЯХ ПЕРЕХОДА СПИНОВОЙ СИСТЕМЫ В СТАЦИОНАРНОЕ СОСТОЯНИЕ ПРИ НАЛИЧИИ ВЫСОКОЧАСТОТНОГО ПОЛЯ
}

Частотные спектры ЯМР жидкостей могут быть получены Фурье-преобразованием сигнала свободной индукции, наведенного в приемной катушке во время различных переходных процессов, протекающих в ядерной спин-системе. Широко распространены методы, основанные на Фурье-преобразовании сигнала, индуцируемого при переходе спин-системы в равновесное состояние во внешнем постоянном магнитном поле. Вывод системы из равновесия может при этом осуществляться самыми различными способами $\left[{ }^{1-6}\right]$.

В настоящей работе теоретически изучаются частотные спектры, полученные Фурье-преобразованием сигнала индукции, регистрируемого в условиях перехода спинсистемы в стационарное состояние в поляризующем и высокочастотном (ВЧ) полях. Рассматриваемый случай охватывает все варианты эксперимента по двойному резонансу с преобразованием Фурье, в которых регистрация сигнала происходит при наличии возмущающего ВЧ-поля, так как импульсное или непрерывное воздействие одного или нескольких ВЧ-полей на систему спинов перед регистрацией можно рассматривать как получение исходного неравновесного состояния.

\section{1. Изменение состояния спин-системы во времени}

По предположению, во время $t>0$ система спинов находится во внешнем магнитном поле

$$
\vec{H}=\vec{k} H_{0}-\overrightarrow{-}-\vec{i} 2 H_{2} \cos \omega_{2} t, \quad H_{0} \gg H_{2} .
$$

$t=0$ является моментом начала регистрации. Состояние спин-системы описывается оператором плотности б, который выбирается в виде суммы трех слагаемых:

$$
\sigma_{T}=\sigma_{0}-1-\chi_{T}^{(s)}+\eta_{T} .
$$

Здесь индекс $т$ обозначает величины, относящиеся к системе координат, вращающейся с частотой $\omega_{2} ; \sigma_{0}$ - больцмановский равновесный оператор плотности $\left[{ }^{7}\right]$ в постоянном мапнитном поле $H_{0} \vec{k} ; \sigma_{0}+\chi_{T}^{(s)}-$ стационарное состояние спин-системы во внешнем поле (1), достигаемое при $t \rightarrow \infty$ и не зависящее от времени во вращающейся системе координат; $\eta_{T}$ - отклонение состояния спин-системы от стационарного. Предполагается, что

$$
\eta_{T}(0)=\chi_{T}^{(0)}-\chi_{T}^{(s)},
$$


т. е. в начальный момент состояние спин-системы может не совпадать с равновесным $\sigma_{0}$.

Для определения состояния спин-системы используем кинетическое уравнение Вангснесса-Блоха-Редфилда (ВБР). Стационарное состояние, очевидно, удовлетворяет уравнению

$$
0=-\left(i \Omega_{2}+\Re\right) \chi_{T}^{(s)}-\mathrm{i} \Omega_{2} \sigma_{0} .
$$

Действие супероператора $\Omega_{2}$ соответствует определению [ $\left.{ }^{7}\right]$

$$
\Omega_{2} \mathbf{O}=\left[\mathbf{H}_{0 T}+\mathbf{H}_{2 T}, \mathbf{O}\right] \text {. }
$$

$\Re$ является редфилдовским супероператором релаксации $\left[{ }^{7}\right]$. Кроме того,

$$
\begin{aligned}
& \mathbf{H}_{0 T}=\mathbf{H}_{0}+\omega_{2} \sum_{i} \mathbf{I}_{z}(i), \\
& \mathbf{H}_{2 T}=\sum_{i} h_{2 i} \mathbf{I}_{x}(i), \\
& h_{2 i}=-\gamma_{i} H_{2},
\end{aligned}
$$

где $\mathbf{H}_{0}$ и $\gamma_{i}$ - гамильтониан спин-системы в магнитном поле $H_{0} \vec{k}\left[{ }^{7}\right]$ и гиромагнитное отношение вместе с химическим сдвигом ядра $i$.

Используя в качестве базиса собственные функции оператора $\mathbf{H}_{0 T}+$ $+\mathbf{H}_{2 T}$ ( $\alpha$-базис), из (4) для недиагональных элементов $\chi_{T}^{(s)}$ получим

$$
\chi_{T \alpha \beta}^{(s)}=-\left[\left(\mathrm{i}_{2}-\Re\right)^{-1} \mathrm{i} \Omega_{2} \sigma_{0}\right]_{\alpha \beta}, \quad \alpha \neq \beta .
$$

В случае достаточно сильного ВЧ-поля $\mathrm{H}_{2}$

$$
\left|\gamma H_{2}\right| \gg\left|\Re_{\alpha \beta \gamma \delta}\right|
$$

и при отсутствии вырожденных уровней у $\mathbf{H}_{0}$ ( $а$-базис)

для

$$
|a-b| \gg\left|\Re_{a b c d}\right|
$$

Следовательно, $\sigma_{0}+\chi_{T}^{(s)}$ является диагональным в $\alpha$-базисе (блоховское приближение).

Диагональные элементы $\chi_{T}^{(s)}$ определяются из системы

$$
\begin{aligned}
& \Re \chi_{T}^{(s)}=0, \\
& \mathrm{Sp} \chi_{T}^{(s)}=0 .
\end{aligned}
$$

Отклонение состояния спин-системы от стационарного изменяется во времени согласно уравнению

$$
\dot{\eta}_{T}=-\left(\mathrm{i} \Omega_{2}-\Re\right) \eta_{T}
$$

С учетом начального условия (3) из последнего вытекает

$$
\eta_{T}=\mathrm{e}^{-t\left(\mathfrak{Q}_{2}-\Re\right)}\left(\chi_{T}^{(0)}-\chi_{T}^{(s)}\right) .
$$

Таким образом, состояние спин-системы во вращающихся координатах изменяется во времени согласно выражению

$$
\sigma_{T}(t)=\sigma_{0}+\chi_{T}^{(s)}+\mathrm{e}^{-t\left(\mathrm{i} \Omega_{2}-\Re\right)}\left(\chi_{T}^{(0)}-\chi_{T}^{(s)}\right) .
$$




\section{2. О наблюдаемом частотном спектре}

Сигнал $S(t)$, индуцируемый в интервале времени от 0 до $T$ ядерной намагниченностью в приемной катушке, направленной по оси $y$ в лабораторной системе координат, регистрируется, умножается на спадающую экспоненту $\mathrm{e}^{-\frac{t}{T_{2}^{*}}}\left(T_{2}^{*}>0\right)$ и подвергается Фурье-преобразованию для получения частотного спектра $S(\omega)$

$$
S(\omega)=\frac{1}{2 \pi} \int_{0}^{T} S(t) \mathrm{e}^{-\frac{t}{T_{2}^{*}}} \mathrm{e}^{-1(\omega t+\varphi)} d t
$$

Знание оператора плотности спин-системы $\sigma_{T}$ позволяет определить сигнал $S(t)$. Действительно, обозначив

$$
\overrightarrow{\mathbf{F}}=\sum_{i} \gamma_{i} \overrightarrow{\mathbf{I}}(i)
$$

имеем

$$
S(t) \sim \frac{d}{d t} \operatorname{Im}\left\{\mathrm{e}^{\mathrm{i} \omega_{2} t} \mathrm{Sp}\left(\sigma_{T} \mathrm{~F}_{-}\right)\right\}
$$

Подстановкой (16) в (19) получаем

$$
\begin{aligned}
& S(t) \sim \operatorname{Im} \operatorname{Sp}\left\{-\left[\mathrm{i}\left(\Omega_{2}-\omega_{2} \mathcal{E}\right)-\Re\right] \mathrm{e}^{-t\left[1\left(\Omega_{2}-\omega \mathcal{E}\right)-\Re\right]}\left(\chi_{T}^{(0)}-\chi_{T}^{(s)}\right) \mathrm{F}_{-}+\right. \\
& \left.+\mathrm{i} \omega_{2} \mathrm{e}^{\mathrm{i} \omega_{2} \mathrm{t}} \chi_{T}^{(s)} \mathbf{F}_{-}\right\} \text {. }
\end{aligned}
$$

Здесь $\mathcal{E}$ - единичный супероператор. Кроме того, в (19) и (20) учтена эрмитовость $\sigma_{T}$, свойство

$$
\mathbf{F}_{+\alpha \beta}^{*}=\mathbf{F}_{-\beta \alpha}
$$

и тот факт, что равновесное состояние $\sigma_{0}$ не может индуцировать сигнал в приемной катушке.

Из (20) видно, что частотный спектр $S(\omega)$ можно представить в виде суммы

$$
S(\omega)=S_{1}(\omega)+S_{2}\left(\omega_{2}\right),
$$

где $S_{1}(\omega)$ и $S_{2}\left(\omega_{2}\right)$ соответствуют двум слагаемым в правой части выражения $(20)$.

При замене верхнего предела интегрирования в (17) на $\infty$ имеем

$\left.S_{2}\left(\omega_{2}\right) \sim I_{2}\left\{\frac{1}{1+\left(\omega_{2}-\omega\right)^{2} T_{2}^{* 2}} \cos \left(\varphi_{2}-\varphi\right)-\frac{\left(\omega_{2}-\omega\right) T_{2}^{*}}{1+\left(\omega_{2}-\omega\right)^{2} T_{2}^{*_{2}}} \sin \left(\varphi_{2}-\varphi\right)\right)_{1}\right\}-$

$-\mathrm{i} I_{2}\left\{\frac{1}{1+\left(\omega_{2}-\omega\right)^{2} T_{2}^{* 2}} \sin \left(\varphi_{2}-\varphi\right)+\frac{\left(\omega_{2}-\omega\right) T_{2}^{*}}{1+\left(\omega_{2}-\omega\right)^{2} T_{2}^{* 2}} \cos \left(\varphi_{2}-\varphi\right)\right\}$.

Такая замена не влечет за собой значительной ошибки в выражении для формы спектра, если только реальный интервал наблюдения $T$ за изменением состояния спин-системы много больше всех временных констант в $(20)$ и $T_{2}{ }^{*}$. Так как в рассматриваемом случае на $T$ не накладывается никаких ограничений, то такая замена вполне обоснованна. Следовательно, $S_{2}\left(\omega_{2}\right)$ содержит одну линию лоренцовой формы с центром в $\omega_{2}$ и полушириной $1 / T_{2}^{*}$. Амплитуда линии 


$$
I_{2}=\omega_{2} T_{2}^{*}\left\{\left[\operatorname{ReSp}\left(\boldsymbol{\chi}_{T}^{(s)} \mathbf{F}_{-}\right)\right]^{2}+\left[\operatorname{ImSp}\left(\chi_{T}^{(s)} \mathbf{F}_{-}\right)\right]^{2}\right\}^{1 / 2}
$$

и фаза

$$
\varphi_{2}=\arctan \frac{\operatorname{ImSp}\left(\chi_{T}^{(s)} \mathbf{F}_{-}\right)}{\operatorname{ReSp}\left(\chi_{T}^{(s)} \mathbf{F}_{-}\right)}
$$

определяются стационарным состоянием спин-системы.

Из (20) и (17) для $S_{1}(\omega)$ получаем следующее выражение:

$$
\begin{aligned}
S_{1}(\omega) \sim & \left(\operatorname { i S p } \left\{[ \mathrm { i } ( \Omega _ { 2 } - \omega _ { 2 } \varepsilon ) - \Re ] \left[\mathrm{i}\left(\Omega_{2}-\omega_{2} \varepsilon+\omega \varepsilon\right)-\right.\right.\right. \\
& \left.\left.\left.-\Re+\frac{1}{T_{2}^{*}} \varepsilon\right]^{-1}\left(\chi_{T}^{(0)}-\chi_{T}^{(s)}\right) F_{-} \mathrm{e}^{-i \varphi}\right\}\right)^{*} .
\end{aligned}
$$

Далее заметим, что в условиях (10) и (11) супероператор i 2 является в первом приближении «диагональным» в $\alpha$-базисе, т. е. он обладает свойством

$$
\left(\mathrm{i} \Omega_{2}-\Re\right)_{\alpha \beta \gamma \delta}=\left(\mathrm{i} \Omega_{\alpha \beta}+\frac{1}{T_{2 \alpha \beta}}\right) \delta_{\alpha \gamma} \delta_{\beta \delta},
$$

где

$$
\begin{aligned}
\Omega_{\alpha \beta} & =\alpha-\beta, \\
\frac{1}{T_{2 \alpha \beta}} & =-\Re_{\alpha \beta \alpha \beta} .
\end{aligned}
$$

Следовательно, супероператоры $\left[\mathrm{i}\left(\Omega_{2}-\omega_{2} \varepsilon\right)-\Re\right]$ и $\left[\mathrm{i}\left(\Omega_{2}-\omega_{2} \varepsilon+\omega \varepsilon\right)-\Re+\frac{1}{T_{2}^{*}} \varepsilon\right]^{-1}$ являются в первом приближении также «диагональными» в $\alpha$-базисе с элементами, соответственно,

$$
\begin{gathered}
{\left[\mathrm{i}\left(\Omega_{2}-\omega_{2} \varepsilon\right)-\Re\right]_{\alpha \beta \gamma \delta}=\left[\mathrm{i}\left(\Omega_{\alpha \beta}-\omega_{2}\right)+\frac{1}{T_{2 \alpha \beta}}\right] \delta_{\alpha \gamma} \delta_{\beta \delta},} \\
\left(\left[\mathrm{i}\left(\Omega_{2}-\omega_{2} \varepsilon+\omega \varepsilon\right)-\Re+\frac{1}{T_{2}^{*}} \varepsilon\right]^{-1}\right)_{\alpha \beta \gamma \delta}=\frac{1}{i\left(\Omega_{\alpha \beta}-\omega_{2}+\omega\right)+\frac{1}{T_{\alpha \beta}}} \delta_{\alpha \gamma} \delta_{\beta \delta} .
\end{gathered}
$$

При этом

$$
\frac{1}{T_{\alpha \beta}}=\frac{1}{T_{2 \alpha \beta}}+\frac{1}{T_{2}^{*}}
$$

С учетом последних выражений для частотного спектра $S_{1}(\omega)$ окончательно получаем

$$
\begin{aligned}
S_{1}(\omega) \sim \sum_{\alpha, \beta} I_{\alpha \beta} & \left\{\frac{1}{1+\left(\Omega_{\alpha \beta}-\omega_{2}+\omega\right)^{2} T_{\alpha \beta}^{2}} \cos \left(\varphi_{\alpha \beta}-\varphi\right)+\right. \\
& \left.+\frac{\left(\Omega_{\alpha \beta}-\omega_{2}+\omega\right) T_{\alpha \beta}}{1+\left(\Omega_{\alpha \beta}-\omega_{2}+\omega\right)^{2} T_{\alpha \beta}^{2}} \sin \left(\varphi_{\alpha \beta}-\varphi\right)\right\}+
\end{aligned}
$$




$$
\begin{aligned}
+\mathrm{i} & \sum_{\alpha, \beta} I_{\alpha \beta}\left\{\frac{1}{1+\left(\Omega_{\alpha \beta}-\omega_{2}+\omega\right)^{2} T_{\alpha \beta}^{2}} \sin \left(\varphi_{\alpha \beta}-\varphi\right)-\right. \\
& \left.-\frac{\left(\Omega_{\alpha \beta}-\omega_{2}+\omega\right) T_{\alpha \beta}}{1+\left(\Omega_{\alpha \beta}-\omega_{2}+\omega\right)^{2} T_{\alpha \beta}^{2}} \cos \left(\varphi_{\alpha \beta}-\varphi\right)\right\} .
\end{aligned}
$$

Учитывая, что

$$
\left|\Omega_{\alpha \beta}-\omega_{2}\right| \gg\left|\Re_{\alpha \beta \gamma \delta}\right|,
$$

пренебрегаем членами, пропорциональными $\Re_{\alpha \beta \gamma \delta}$. Тогда амплитуды и фазы отдельных спектральных линий определятся следующими выражениями:

$$
\begin{gathered}
I_{\alpha \beta}=\left(\Omega_{\alpha \beta}-\omega_{2}\right) T_{\alpha \beta}\left\{\left(\operatorname{Re}\left[\left(\chi_{T}^{(0)}-\chi_{T}^{(s)}\right)_{\alpha \beta} \mathbf{F}_{-\beta \alpha}\right]\right)^{2}+\right. \\
\left.+\left(\operatorname{Im}\left[\left(\chi_{T}^{(0)}-\chi_{T}^{(s)}\right)_{\alpha \beta} \mathbf{F}_{-\beta \alpha}\right]\right)^{2}\right\}^{1 / 2} \\
\varphi_{\alpha \beta}=\arctan \frac{\operatorname{Im}\left[\left(\chi_{T}^{(0)}-\chi_{T}^{(s)}\right)_{\alpha \beta} \mathbf{F}_{-\beta \alpha}\right]}{\operatorname{Re}\left[\left(\chi_{T}^{(0)}-\chi_{T}^{(s)}\right)_{\alpha \beta} \mathbf{F}_{-\beta \alpha}\right]} .
\end{gathered}
$$

На основе полученных выражений можно сделать некоторые общие выводы о частотном спектре, наблюдаемом в случае перехода спинсистемы в стационарное состояние при наличии возмущающего ВЧ-поля.

Во-первых, в спектре наблюдается линия на частоте возмущающего ВЧ-поля. Эта линия является составной, так как определенный вклад в нее, наряду с линией спектра $S_{2}\left(\omega_{2}\right)$ (23), могут вносить и компоненты спектра $S_{1}(\omega)$ (33) с совпадающими индексами $\alpha=\beta$. Вследствие этого форма линии может отклоняться от лоренцовой.

Во-вторых, любая пара уровней $\alpha$-базиса, в принципе, дает в частотном спектре $S_{1}(\omega)$ две линии, расположенные симметрично относительно частоты возмущающего поля

$$
\omega=\omega_{2} \pm \Omega_{\alpha \beta} .
$$

Полуширины этих линий зависят как от релаксационных коэффициентов в $\alpha$-базисе, так и от параметра $T_{2}^{*}$. Амплитуды линий, соответствующих одному переходу $\alpha$-базиса, могут и не быть равными. Максимальное количество наблюдаемых линий (и их происхождение в смысле производящего перехода) в данном эксперименте определяется количеством (и положением) ненулевых недиагональных элементов оператора $\mathbf{F}_{-}$в $\alpha$-базисе, т. е. зависит от частоты и напряженности возмущающего ВЧполя. Реально наблюдаемое количество линий зависит, кроме того, и от наличия соответствующего ненулевого элемента оператора $\chi_{T}^{(0)}-\chi_{T}^{(s)}$ в $\alpha$-базисе, т. е. от способа подготовки состояния спин-системы в эксперименте. Любая линия в спектре $S_{1}(\omega)$ может быть приведена к чистой лоренцовой форме поглощения или дисперсии.

Таким образом, наблюдаемый частотный спектр во многом напоминает спектры двойного резонанса с сильным возмущением $\left[{ }^{8}\right]$. Они могут различаться по количеству наблюдаемых линий и по их амплитудам, зависящим от конкретного способа подготовки состояния спинсистемы. Эти величины, как и положения центров линий, должны определяться отдельно в каждом частном случае. 


\section{3. Система $A X$}

Ниже рассматривается частный случай системы $A X$, состоящей из двух спинов $1 \frac{2}{2}$. При этом предполагается, что в начальный момент спинсистема находится в равновесном состоянии во внешнем постоянном магнитном поле

$$
\chi^{(0)}=0 .
$$

За исходный базис для записи операторов принимается $a$-базис. Оказывается, однако, что получить преобразование, связывающее $а$-базис с $\alpha$-базисом, в общем случае в аналитическом виде не удается. Поэтому необходимо рассматривать различные варианты эксперимента в отдельности.

1. ВЧ-поле воздействует непосредственно только на один переход. Это означает выполнение условий

$$
|\delta| \lesssim\left|h_{2 i}\right| \ll\left|\delta_{A}\right|,|I| .
$$

Здесь

$$
\delta_{x}=\frac{1}{2} I+\delta, \quad \delta_{i}=\omega_{0 i}+\omega_{2}, \quad I=2 \pi J,
$$

а $\omega_{0 i}$ и $J$ имеют общепринятый смысл. Условие (10) и вытекающее из него следствие (12) считаются, однако, выполненными.

Далее, учитывая неравенства (39), пренебрегаем в матрице $\mathbf{H}_{0 T}+\mathbf{H}_{2 T}$ всеми недиагональными элементами $\left(\mathbf{H}_{0 T}+\mathbf{H}_{2 T}\right)_{a b}$, у которых абсолютная величина разности соответствующих диагональных элементов $\left|\left(\mathbf{H}_{0 T}+\mathbf{H}_{2 T}\right)_{a a}-\left(\mathbf{H}_{0 T}+\mathbf{H}_{2 T}\right)_{b b}\right|$ порядка $\left|\delta_{A}\right|$ или $|I|$. Полученная приближенная матрица диагонализируется и определяется преобразование, связывающее $a$-базис с приближенным $\alpha$-базисом.

Из матрицы оператора $\mathbf{F}_{-}$в $\alpha$-базисе следует, что в данном случае максимально возможное число $A$-линий в спектре $S_{1}(\omega)$ равно четырем (по одной на каждый из переходов $1-3,1-4,2-3,2-4$ оператора $\mathbf{H}_{0 T}+\mathbf{H}_{2 T}$ в $\alpha$-базисе), а $X$-линий - трем (одна из которых соответствует переходу $1-2$, и две - переходу $3-4)$. Однако в рассматриваемом конкретном эксперименте (38) в спектре наблюдаются только две $X$-линии, обязанные переходу 3 -4. Линии расположены симметрично относительно $\omega_{2}$ на частотах

$$
\omega^{(i)}=\omega_{2} \pm \sqrt{\delta^{2}+h_{2 x}^{2}}, \quad i=1,2,
$$

и направлены в разные стороны от нулевой линии; амплитуды их различны и определяются как

$$
I^{(i)}= \pm \frac{1}{4} \omega^{(i)}\left(\omega_{0 x}-\frac{1}{2} I\right) q \gamma_{x} T_{34} \sin \alpha(1 \mp \cos \alpha), \quad i=1,2,
$$

где

$$
\alpha=\arctan \frac{h_{2 x}}{\delta} .
$$

Фазы обеих линий совпадают $\left(\varphi_{34}=\varphi_{43}\right)$.

2. ВЧ-поле непосредственно воздействует на переходы только одного ядра:

$$
\left|\delta_{A}\right| \gg\left|\delta_{x}\right|,\left|h_{2 i}\right| .
$$

В настоящем случае приближенный $\alpha$-базис и преобразование, связывающее его с $а$-базисом, определяются, как и в предыдущем случае, 
посредством пренебрежения на основе (44) в матрице $\mathbf{H}_{0 T}+\mathbf{H}_{2 T}$ частью недиагональных элементов, поскольку разность им соответствующих диагональных элементов порядка $\left|\delta_{A}\right|$.

Благодаря ненулевым недиагональным элементам матрицы $F_{-}$в $\alpha$ базисе в спектре могут появиться на отличных от $\omega_{2}$ частотах как максимум четыре $A$-линии - по одной на переходы $1-3,1-4,2-3,2-4$ и четыре $X$-линии - по две на переходы $1-2,3-4$. В рассматриваемом варианте (38) в спектре появляются из них только четыре $X$-линии. Центры их расположены попарно-симметрично относительно $\omega_{2}$

$$
\begin{aligned}
& \omega^{(i)}=\omega_{2} \mp \sqrt{\left(\delta_{x}+\frac{1}{2} I\right)^{2}+h_{2 x}^{2}}, \quad i=1,2, \\
& \omega^{(i)}=\omega_{2} \mp \sqrt{\left(\delta_{x}-\frac{1}{2} I\right)^{2}+h_{2 x}^{2}}, \quad i=3,4 .
\end{aligned}
$$

Линии одной пары направлены в разные стороны от нулевой линии и амплитуды их различны:

$$
\begin{aligned}
& I^{(i)}=\mp \frac{1}{4} \omega^{(i)}\left(\omega_{0 x}+\frac{1}{2} I\right) q_{\gamma_{x}} T_{12} \sin \varphi(1 \pm \cos \varphi), \quad i=1,2, \\
& I^{(i)}=\mp \frac{1}{4} \omega^{(i)}\left(\omega_{0 x}-\frac{1}{2} I\right) q_{\gamma_{x}} T_{34} \sin \vartheta(1 \pm \cos \vartheta), \quad i=3,4 .
\end{aligned}
$$

Здесь верхние знаки относятся к нечетным значениям $i$, а нижние - к четным;

$$
\varphi=\arctan \frac{h_{2 x}}{\delta_{x}+\frac{1}{2} I}, \quad \vartheta=\arctan \frac{h_{2 x}}{\delta_{x}-\frac{1}{2} I} .
$$

Фазы всех линий также совпадают.

В случае точной настройки, $\delta_{x}=0$, или достаточно сильного возмущающего ВЧ-поля,

$$
\left|h_{2 x}\right| \gg\left|\delta_{x} \pm \frac{1}{2} I\right|
$$

происходит коллапс линий 1,3 и 2,4. При выполнении последнего условия амплитуды линий одной пары также становятся равными.

3. ВЧ-поле воздействует на переходы обоих ядер:

$$
\left|h_{2 i}\right| \gg\left|\delta_{i}\right|,|I| \text {. }
$$

Для определения приближенного $\alpha$-базиса в настоящем случае сначала диагонализируется $\mathbf{H}_{0 T}+\mathbf{H}_{2 T}$ относительно $h_{2 i}$. После этого производится приближение: в преобразованной матрице $\mathbf{H}_{0 T}+\mathbf{H}_{2 T}$ пренебрегается частью недиагональных элементов, поскольку разность им соответствующих диагональных элементов порядка $\left|h_{2 i}\right|$. Полученная матрица затем диагонализируется и определяется приближенный $\alpha$ базис.

В данном случае отличаются от нуля все недиагональные элементы матрицы оператора $\mathbf{F}_{-}$в $\alpha$-базисе, за исключением $\mathbf{F}_{-14}$ и $\mathbf{F}_{-41}$. Следовательно, в спектре можно ожидать появления десяти линий на частотах, отличных от $\omega_{2}$ (две на каждый переход, за исключением перехода 
$1-4)$, которые уже нельзя разделить на $A$ - и $X$-линии. Однако так как частоты переходов $1-2$ и $3-4$, а также переходов $1-3$ и $2-4$ совпадают, то максимальное число линий в спектре равно шести. При этом четыре из них являются составными.

В принципе, при выполнении (38) в спектре наблюдаются все шесть линий. Однако, амплитуды тех из них, которые появляются на частотах

$$
\omega=\omega_{2} \mp \sqrt{\left(h_{2 A}-h_{2 x}\right)^{2}+\frac{1}{4} I^{2}}
$$

и соответствуют переходу $2-3$, относятся к амплитудам остальных линий приблизительно как $\left|I\left(\gamma_{A}-\gamma_{x}\right)\right|:\left|\omega_{0 i} \gamma_{i}\right|$. Остальные четыре составные линии появляются на частотах

$$
\begin{aligned}
& \omega^{(i)}=\omega_{2} \mp \frac{1}{2}\left[\left(h_{2 A}+h_{2 x}\right)+\sqrt{\left(h_{2 A}-h_{2 x}\right)^{2}+\frac{1}{4} I^{2}}\right], \quad i=1,2, \\
& \omega^{(i)}=\omega_{2} \mp \frac{1}{2}\left[\left(h_{2 A}+h_{2 x}\right)-\sqrt{\left(h_{2 A}-h_{2 x}\right)^{2}+\frac{1}{4} I^{2}}\right], \quad i=3,4 .
\end{aligned}
$$

Так как фазы всех компонентов одинаковы, то наблюдаемые амплитуды составных линий являются суммами амплитуд составляющих компонентов.

Упрощенные выражения для амплитуд наблюдаемых составных линий следующие:

$$
\begin{array}{r}
I^{(i)}=\mp \frac{1}{8} \omega^{(i)} q\left(T_{13}+T_{24}\right)\left[\omega_{0 A} \gamma_{A}(1+\cos \beta)+\omega_{0 x} \gamma_{x}(1-\cos \beta)\right], \\
i=1,2 \\
I^{(i)}=\mp \frac{1}{8} \omega^{(i)} q\left(T_{12}+T_{34}\right)\left[\omega_{0 A} \gamma_{A}(1-\cos \beta)+\omega_{0 x} \gamma_{x}(1+\cos \beta)\right], \\
i=3,4 .
\end{array}
$$

При этом

$$
\beta=\arctan \frac{\frac{1}{2} I}{h_{2 A}-h_{2 x}} .
$$

Выражения (55) и (56) действительны, если естественные ширины линий в $\alpha$-базисе отличаются друг от друга незначительно

или же

$$
\left|\Re_{\alpha \beta \alpha \beta}-\Re_{\gamma \delta \gamma \delta}\right| \ll\left|\Re_{\alpha \beta \alpha \beta}+\Re_{\gamma \delta \delta \delta}\right|,
$$

$$
T_{2}^{*} \ll T_{2 \alpha \beta}
$$

Линии в парах $i=1,2$ и $i=3,4$ имеют приблизительно одинаковые амплитуды и направлены в разные стороны от нулевой линии. Если $\left|h_{2 A}-h_{2 x}\right| \ll\left|\frac{1}{2} I\right|, \quad$ то амплитуды всех линий приблизительно равны. В противном случае можно говорить о линиях $A$ и $X$, а систему $A X$ рассматривать как систему из двух несвязанных спинов. 


\section{Заключение}

Кинетическое уравнение ВБР использовано для изучения частотного спектра, полученного Фурье-преобразованием сигнала, индуцированного в приемной катушке во время перехода спин-системы в стационарное состояние при наличии возмущающего ВЧ-поля.

Оказалось, что наблюдаемый спектр во многом напоминает спектр двойного резонанса с сильным возмущением. Положения появляющихся линий определяются уровнями гамильтониана, не зависящего от времени во вращающейся с частотой $\omega_{2}$ системе координат. Количество и амплитуды линий зависят от матричных элементов оператора $\mathbf{F}_{-}$в $\alpha$ базисе, т. е. от частоты и напряженности возмущающего ВЧ-поля, а также от состояния спин-системы к моменту начала регистрации переходного процесса. Наблюдаемые линии состоят из компонентов лоренцовой формы, их естественные полуширины определяются релаксационными коэффициентами в $\alpha$-базисе.

Полученные выводы могут быть использованы для объяснения экспериментов по различным мультирезонансам с преобразованием Фурье, в которых регистрация сигнала производится при наличии одного ВЧвозмущения. Разные манипуляции над спин-системой до регистрации сводятся к подготовке начального состояния спин-системы и учитываются оператором плотности $\chi^{(0)}$.

Теория иллюстрируется на примере системы $A X$ из двух спинов $1 / 2$.

\section{Л ИТЕРАТ У Р А}

1. Ernst R. R., Anders on W. A., Rev. Sci. Instr., 37, 93 (1966).

2. Vold R. L., W a ugh J. S., Kle in M. P., Phelps D. E., J. Chem. Phys., 48, 3831 (1968).

3. Freem a n R., Hill H. D. W., J. Chem. Phys,, 53, 4103 (1970).

4. Freem an R., Hill H. D. W., J. Chem. Phys., 54, 3367 (1971).

5. Lalanne P., Andrieux A., Lemanceau B., Lussan C., Ber. Bunsenges. phys. Chem., 75, 275 (1971).

6. Solom on I., C. R. Acad. Sci. Paris, 248, 92 (1959).

7. К ундл а Э., Изв. АН ЭССР, Физ. Матем., 22, 267 (1973).

8. К ундл а Э., Сини и еэ В., Изв. АН ЭССР, Физ. Матем., 18, 276 (1969).

Ннститут кибернетики

Академии наук Эстонской ССР
Поступила в редакцию 26/V 1975

\section{E. KUNDLA}

\section{RAADIOSAGEDUSVÄLJA TOIMEL TEKKIVASSE STATSIONAARSESSE SEISUNDISSE SIIRDUVATE VEDELIKE TMR FOURIER' SPEKTRITEST}

Uuritakse vedelike TMR sagedusspektreid, mis on saadud spinnsüsteemide meelevaldsest lähteseisundist raadiosagedusvälja ja tugeva alalismagnetvälja toimel tekkivasse statsionaarsesse seisundisse ülemineku korral indutseeritud signaali Fourier' teisenduse kaudu. Ilmneb, et saadavad spektrid sarnanevad tugeva raadiosagedusväljaga teostatud topeltresonantsi spektritega. Joonte asendid on määratud raadiosagedusvälja sagedusega pöörlevates koordinaatides ajast sōltumatu hamiltoniaani omaväärtustega. Joonte arv ja nende amplituudid sōltuvad operaatori $\mathbf{F}_{-}=\sum_{i} \gamma_{i} \mathbf{I}_{-}(i)$ maatrikselementidest eelnimetatud hamiltoniaani omafunktsioonide baasil, seega raadiosagedusvälja sagedusest ja tugevusest, aga ka spinnsüsteemi seisundist signaali registreerimise alghetkel. Teooriat rakendatakse kahest poolarvulisest spinnist koosneva $A X$-süsteemi puhul. 


\section{E. KUNDLA}

\section{ON THE FOURIER TRANSFORM NMR SPECTRUM OF LIQUIDS OBTAINED BY TRANSITION OF THE SPIN SYSTEM TO A STATIONARY STATE IN THE PRESENCE OF A RADIOFREQUENCY FIELD}

The NMR frequency spectrum of a liquid obtained by Fourier Transform of the signal registered by transition of a spin system from any state to the stationary state in the presence of a radiofrequency (r. f.) and static magnetic field is investigated. It appears that the mentioned spectra are quite similar to the double resonance spectra with a strong disturbing $r$. f. field. The centres of the lines are determined by the eigenvalues of the hamiltonian which is independent of time in the rotating coordinate frame. The number of lines and their amplitudes are determined by the matrix elements of the operator $\mathbf{F}_{-}=\sum_{i} \gamma_{i} \mathbf{I}_{-}(i)$ on the basis of eigenfunctions of the aforementioned hamiltonian, i.e. on the frequency and intensity of the r.f. field, and on the state of the spin system at the beginning of the registration. The theory is illustrated by an $A X$ system. 